\title{
Impact of COVID-19 pandemic on psychophysical stress in patients with adrenal insufficiency: the CORTI-COVID study
}

\author{
M. Martino ${ }^{1}$ (1) $\cdot$ N. Aboud ${ }^{1} \cdot$ M. F. Cola ${ }^{1} \cdot$ G. Giancola ${ }^{1} \cdot$ A. Ciarloni $^{1} \cdot$ G. Salvio ${ }^{1} \cdot$ G. Arnaldi $^{1}$ (]
}

Received: 14 August 2020 / Accepted: 8 September 2020 / Published online: 18 September 2020

(C) Italian Society of Endocrinology (SIE) 2020

\begin{abstract}
Purpose COVID-19 is a novel threat to patients with adrenal insufficiency (AI), whose life expectancy and quality (QoL) are impaired by an increased risk of infections and stress-triggered adrenal crises (AC). If infected, AI patients require prompt replacement tailoring. We assessed, in a cohort of AI patients: prevalence and clinical presentation of COVID-19; prevalence of AC and association with intercurrent COVID-19 or pandemic-related psychophysical stress; lockdown-induced emotional burden, and health-related QoL.

Methods In this monocentric (Ancona University Hospital, Italy), cross-sectional study covering February-April 2020, 121 (40 primary, 81 secondary) AI patients (59 males, $55 \pm 17$ years) completed telematically three questionnaires: the purposebuilt "CORTI-COVID", assessing medical history and concern for COVID-19-related global health, AI-specific personal health, occupational, economic, and social consequences; the AddiQoL-30; the Short-Form-36 (SF-36) Health Survey.

Results COVID-19 occurred in one (0.8\% prevalence) 48 -year-old woman with primary AI, who promptly tailored her replacement. Dyspnea lasted three days, without requiring hospitalization. Secondary AI patients were not involved. No AC were experienced, but pandemic-related stress accounted for $6 / 14$ glucocorticoid up-titrations. Mean CORTI-COVID was similar between groups, mainly depending on "personal health" in primary $\mathrm{AI}(\rho=0.888, p<0.0001)$ and "economy" in secondary AI $(\rho=0.854, p<0.0001)$. Working restrictions increased occupational concern. CORTI-COVID correlated inversely with QoL. AddiQoL-30 and SF-36 correlated strongly. Comorbidities worsened patients' QoL.

Conclusion If educational efforts are made in preventing acute events, AI patients seem not particularly susceptible to COVID-19. The novel "CORTI-COVID" questionnaire reliably assesses the pandemic-related emotional burden in AI. Even under unconventional stress, educated AI patients preserve a good QoL.
\end{abstract}

Keywords SARS-CoV-2 $\cdot$ Adrenal crisis $\cdot$ Addison $\cdot$ Hypopituitarism $\cdot$ AddiQoL $\cdot$ Telemedicine COVID

\section{Introduction}

In Adrenal insufficiency (AI), the adrenal cortex is unable to produce adequate amounts of glucocorticoids with respect to the body's needs. AI can result either from a direct damage

N. Aboud and M. F. Cola contributed equally to the manuscript.

Electronic supplementary material The online version of this article (https://doi.org/10.1007/s40618-020-01422-2) contains supplementary material, which is available to authorized users.

G. Arnaldi

g.arnaldi@staff.univpm.it

1 Department of Endocrinology and Metabolic Diseases (DISCLIMO), Polytechnic University of Marche, Via Conca 71, 60126 Ancona, Italy or impaired function of the adrenal gland (primary AI), or from the indirect impairment of adrenal function which occurs in ACTH deficiency (secondary AI) [1,2].

Since adrenal hormones are crucial in preserving energy, fluid and salt homeostasis, as well as in modulating stress response and immune system, AI is a severe, potentially life-threatening condition [1]. Despite optimal replacement, indeed, AI patients experience higher morbidity and mortality rates than the general population, a consistent reduction in life expectancy, and notable impairment in self-reported quality of life $(\mathrm{QoL})$ and health status, regardless of AI etiology and comorbidities [3-5].

Tailored therapeutic regimens and educational measures aimed at preventing adrenal crises are key points in the management of chronic AI [6]. Nevertheless, more than $10 \%$ of AI patients followed-up for two years undergoes an acute 
event, which is fatal in $6 \%$ and triggered by intercurrent infections or emotional stressors [7].

Recently, Italy has been overwhelmed by COVID-19 pandemic due to novel Coronavirus SARS-CoV-2, which clinical features are fever, dry cough, dyspnea and, in worst cases, severe/critical pneumonia with acute respiratory distress syndrome (ARDS) requiring intensive care management, acute kidney failure, and death [8-12]. Lockdown measures were disposed throughout the country to contain the spreading of the infection through social distancing and the blockade of non-essential productive activities, with unavoidable social and economic repercussions.

To date, expert opinions on COVID-19 stage-specific adjustment of glucocorticoid replacement in AI are available $[13,14]$. However, there are no data on the impact of SARSCoV-2 pandemic in AI patients, nor about psychophysical stress resulting from their awareness of being more susceptible to the infection and at risk of adrenal crises in a context of social isolation, uncertainty, and concern about the future.

$\mathrm{We}$, therefore, aimed to investigate in a cohort of AI patients, for the period February-April 2020: (a) the prevalence, clinical features, and outcome of COVID-19; (b) the prevalence of adrenal crises and their possible association with intercurrent infection with SARS-CoV-2 and/or pandemic-related psychophysical stress; (c) the emotional impact of pandemic-induced lockdown and social distancing; (d) the self-reported QoL and health perception during the lockdown.

\section{Methods}

\section{Study design}

This was an open-label, cross-sectional study conducted in April 2020 on a cohort of AI patients regularly followed-up at the Endocrinology and Metabolic Diseases Department of Ancona University Hospital, Ancona, Italy. The study was conducted according to the Declaration of Helsinki and approved by the local Ethic Committee.

\section{Patients and materials}

Due to the current lockdown measures, 202 outpatients with a history of primary or secondary AI, defined according to the latest Endocrine Society Guidelines criteria [1, 2], were contacted by a phone call or email. Of them, 81 were discarded due to unavailability $(n=29)$, lack of consent $(n=35)$, transitory AI $(n=7)$, and death $(n=10$; Fig. 1). After obtaining the informed consent, which was

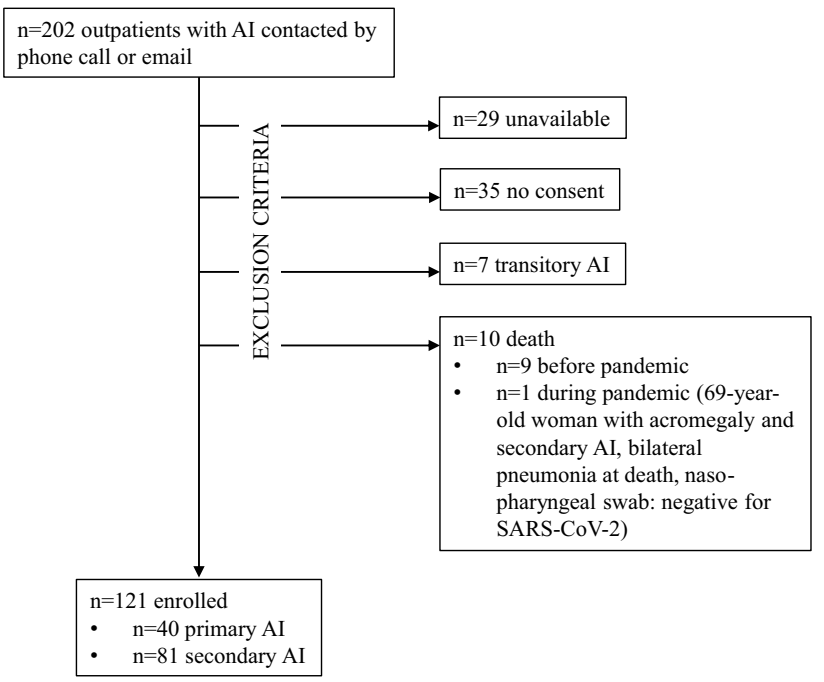

Fig. 1 Patient flow diagram

given by a parent/legal guardian for patients $<18$ years, the remaining 121 (59 males and 62 females, 40 primary $\mathrm{AI}$ and 81 secondary $\mathrm{AI}$ ) were enrolled and underwent the following three-section interview (Fig. 2):

1 The purpose-built "CORTI-COVID" questionnaire, composed by 34 questions and four issues: demography and anthropometric data, general and AI-specific medical and pharmacological history, COVID-19-specific familiar (closer contacts included), physiological and medical history, emotional impact of COVID-19. Quantification of this last issue in the "CORTI-COVID score" expressed the degree of each participant's concern for the pandemic. The CORTI-COVID score (minimum 5 , maximum 25 points, a higher score indicating higher concern) was calculated as the sum of the scores obtained from five questions, which explored the degree of concern for COVID-19-related global health, personal health as an AI patient, occupational consequences, economic impact and social implications (minimum 1, maximum 5 points each);

2 The AI-specific self-reported QoL questionnaire "AddiQoL-30" [15-17];

3 The self-reported health perception questionnaire "Short-Form-36 Health Survey (SF-36)" [18].

The results of the interview were analyzed in the whole cohort at first, then subgroup analyses were conducted according to AI etiology (primary vs secondary). Demography, glucocorticoid replacement and 


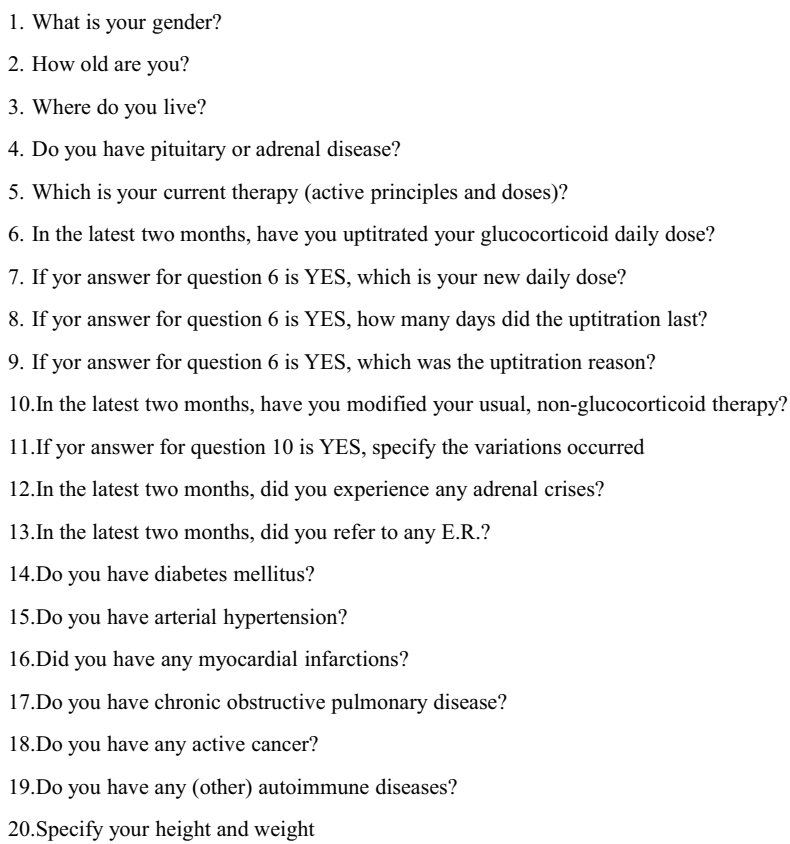

Fig. 2 The "CORTI-COVID" questionnaire

comorbidities impacting COVID-19 prognosis were also considered.

\section{Statistical analysis}

Variables are presented as mean \pm standard deviation (SD) if continuous, number and percentage if categorical, mean and corresponding 95\% confidence interval (CI) if ordinal. After verifying the normal distribution of quantitative variables with the Kolmogorov-Smirnov test, comparisons between two groups were made with the Student's $t$ test (normal distribution) or the Mann-Whitney test (non-normal distribution). For comparisons concerning more than two groups, the ANOVA (parametric) or the Kruskal-Wallis (non-parametric) tests were applied. Categorical variables were compared with the Chi-square $\left(\chi^{2}\right)$ or the Fisher's exact test, where appropriate. The Spearman's Rho $(\rho)$ was used for the bi-varied correlation analyses. A $p$ value of 0.050 was considered for statistical significance. The SPSS (SPSS Inc, Chicago, IL, USA) software version 20.0 was used for statistical analysis.

\section{Results}

Table 1 illustrates patients' demographic and clinical features. Primary AI patients were younger $(p=0.033)$ and mostly females $(p=0.002)$; geographical distribution was homogeneous between subgroups $(p>0.050)$. Distribution of AI etiologies is shown in Fig. 3. Among secondary AI

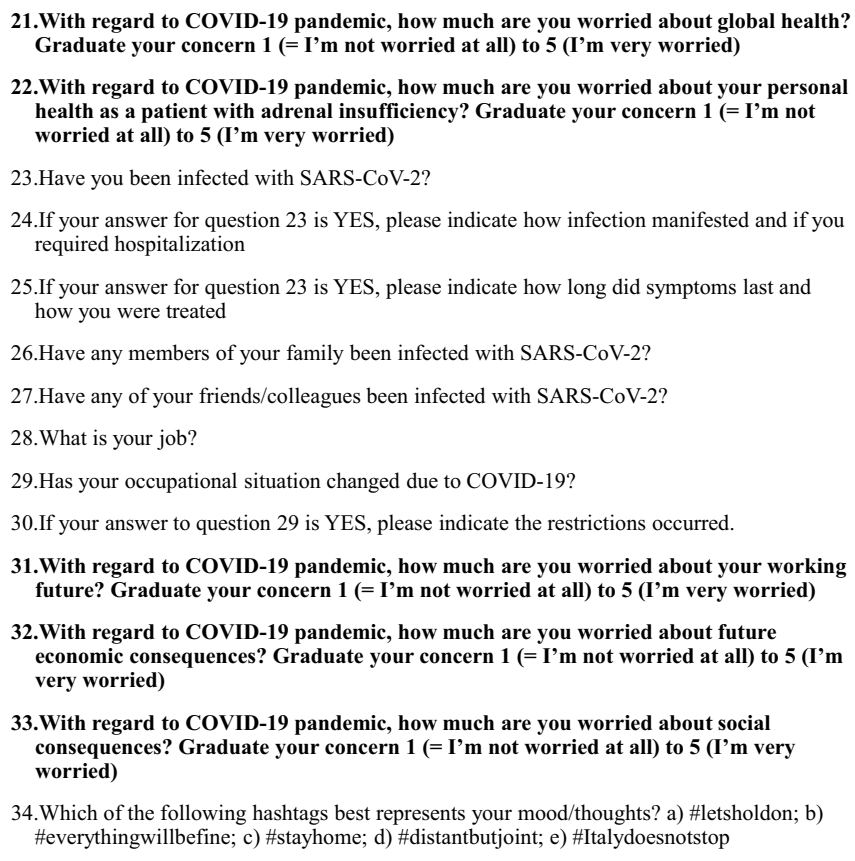

33.With regard to COVID-19 pandemic, how much are you worried about social consequences? Graduate your concern 1 (= I'm not worried at all) to 5 (I'm very worried)

34. Which of the following hashtags best represents your mood/thoughts? a) \#letsholdon; b) \#everythingwillbefine; c) \#stayhome; d) \#distantbutjoint; e) \#Italydoesnotstop

patients, eleven (13.6\%) had isolated ACTH deficiency; in the others, secondary AI was variably associated to TSH (82.7\%), FSH/LH (50.6\%), GH (34.6\%), and argininevasopressin (AVP, 30.9\%) deficiencies, which were adequately replaced, when necessary. Among the comorbidities impacting COVID-19 prognosis, such as diabetes mellitus, hypertension and cardiovascular diseases, obesity, chronic obstructive pulmonary disease (COPD), active cancer, and autoimmune disorders, only hypertension and autoimmune disorders were differently prevalent between groups. Of note, in secondary AI patients there was a significant gender difference concerning the presence of overweight/obesity (34 males and 12 females, $p<0.0001$ ) and hypertension (20 males and seven females, $p=0.020$ ). Hypertensive patients with secondary AI had a higher Body Mass Index (BMI $28.2 \pm 5.2$ vs $\left.25.7 \pm 4.2 \mathrm{~kg} / \mathrm{m}^{2}, p=0.030\right)$ and were older ( $66 \pm 11$ vs $55 \pm 18$ years, $p=0.003$ ) than normotensives.

\section{CORTI-COVID}

Total and partial CORTI-COVID scores are shown in Table 2. Concern for economy most influenced the final score $(\rho=0.816, p<0.0001)$; however, all items moderately (occupational and social concern) or strongly (global and personal health concern) correlated with total CORTICOVID score.

All CORTI-COVID scores were similar between primary and secondary AI patients, without gender differences. Elder participants showed a lower occupational concern (correlation with age $\rho=-0.388, p<0.0001$ ). Hypertensive 
Table 1 Demographic and clinical features of study participants

\begin{tabular}{|c|c|c|c|c|}
\hline & All $(n=121)$ & Primary AI $(n=40)$ & Secondary AI $(n=81)$ & $p$ value \\
\hline Gender & & & & 0.033 \\
\hline Male & $59(48.8)$ & $14(35.0)$ & $45(55.6)$ & \\
\hline Female & $62(51.2)$ & $26(65.0)$ & $36(44.4)$ & \\
\hline Age (years) & $55.3 \pm 17.0(15.0-84.0)$ & $49.0 \pm 15.8(15.0-83.0)$ & $58.5 \pm 16.8(18.0-84.0)$ & 0.002 \\
\hline Weight (kg) & $73.4 \pm 17.0(44.0-140.0)$ & $68.3 \pm 13.2(49.0-95.0)$ & $75.9 \pm 18.2(44.0-140.0)$ & 0.026 \\
\hline BMI $\left(\mathrm{kg} / \mathrm{m}^{2}\right)$ & $26.2 \pm 4.5(18.8-41.8)$ & $25.5 \pm 4.0(18.8-34.9)$ & $26.6 \pm 4.8(19.0-41.8)$ & NS \\
\hline Glucocorticoid replacement & & & & $<0.0001$ \\
\hline $\mathrm{CA}$ & $52(43.0)$ & $7(17.5)$ & $45(55.6)$ & \\
\hline $\mathrm{HC}$ & $65(53.7)$ & $32(80.0)$ & $33(40.7)$ & \\
\hline PRED & $3(2.5)$ & 0 & $3(3.7)$ & \\
\hline DEX & $1(0.8)$ & $1(2.5)$ & 0 & \\
\hline Glucocorticoid dose (mg HC/day) & $18.7 \pm 7.2(5.0-35.0)$ & $20.8 \pm 7.2(5.0-35.0)$ & $17.7 \pm 7.0(5.0-35.0)$ & 0.030 \\
\hline Lockdown stress dose & & & & NS \\
\hline Yes & $14(11.6)$ & $6(15.0 \%)$ & $8(9.9)$ & \\
\hline No & $107(88.4)$ & $34(85.0 \%)$ & $73(90.1)$ & \\
\hline Stress dose (mg HC/day) & $20.7 \pm 33.9(2.5-100.0)$ & $23.3 \pm 38.0(5.0-100.0)$ & $18.8 \pm 33.1(5.0-100.0)$ & NS \\
\hline Stress dose duration (weeks) & & & & NS \\
\hline$<1$ & $6(42.9)$ & $4(66.7)$ & $2(25.0)$ & \\
\hline $1-2$ & $2(14.3)$ & 0 & $2(25.0)$ & \\
\hline $2-3$ & 0 & 0 & 0 & \\
\hline $3-4$ & $2(14.3)$ & $1(16.7)$ & $1(12.5)$ & \\
\hline$>4$ & $2(14.3)$ & $1(16.7)$ & $1(12.5)$ & \\
\hline Not known & $2(14.3)$ & 0 & $2(25.0)$ & \\
\hline Mineralocorticoid replacement & & & & NA \\
\hline Yes & NA & $36(90)$ & NA & \\
\hline No & NA & $4(10)$ & NA & \\
\hline \multicolumn{5}{|l|}{ Comorbidities } \\
\hline Diabetes mellitus & $10(8.3)$ & $4(10)$ & $6(7.4)$ & NS \\
\hline Hypertension & $33(27.3)$ & $6(15)$ & $27(33.3)$ & 0.033 \\
\hline Overweight/obesity & $65(53.7)$ & $19(47.5)$ & $46(56.8)$ & NS \\
\hline Ischemic heart disease & $3(2.5)$ & $1(2.5)$ & $2(2.5)$ & NS \\
\hline COPD & $10(8.3)$ & $4(10)$ & $6(7.4)$ & NS \\
\hline Active cancer & $2(1.7)$ & $1(2.5)$ & $1(1.2)$ & NS \\
\hline (Other) autoimmune diseases & $29(24.0)$ & $20(50)$ & $9(11.1)$ & $<0.0001$ \\
\hline
\end{tabular}

Data are expressed as number and percentage or as mean \pm SD (minimum-maximum range)

$B M I$ Body Mass Index, $C A$ cortisone acetate, $D E X$ dexamethasone, $H C$ hydrocortisone, $P R E D$ prednisone, $C O P D$ chronic obstructive pulmonary disease, $N S$ not statistically significant, $N A$ not applicable

patients were more worried for COVID-19-related global health $(p=0.005)$ and less for occupational consequences $(p=0.003)$ than normotensives; if a history of ischemic heart disease was present, concern for both global and personal health was higher $(p=0.043)$. Similarly, people who suffered from autoimmune diseases were more afraid of personal health consequences than those who did not $(p=0.038)$.

Patients who required replacement adjustments were 14 ( 6 with primary AI, 8 with secondary AI). No adrenal crises were experienced, but COVID-19-related emotional stress accounted for 6 of 14 glucocorticoid stress doses ( 5 women with primary AI, 1 man with secondary AI). All CORTICOVID results were similar between patients who required glucocorticoid stress doses and those who did not; also, no significant correlations were found between the scores and patients' glucocorticoid daily dose. Compared to the others, patients who adjusted their glucocorticoid dose due to COVID-19-related emotional stress had similar gender, age, AI etiology distribution, used overlapping replacement doses, and did not differ with respect to comorbidities, 

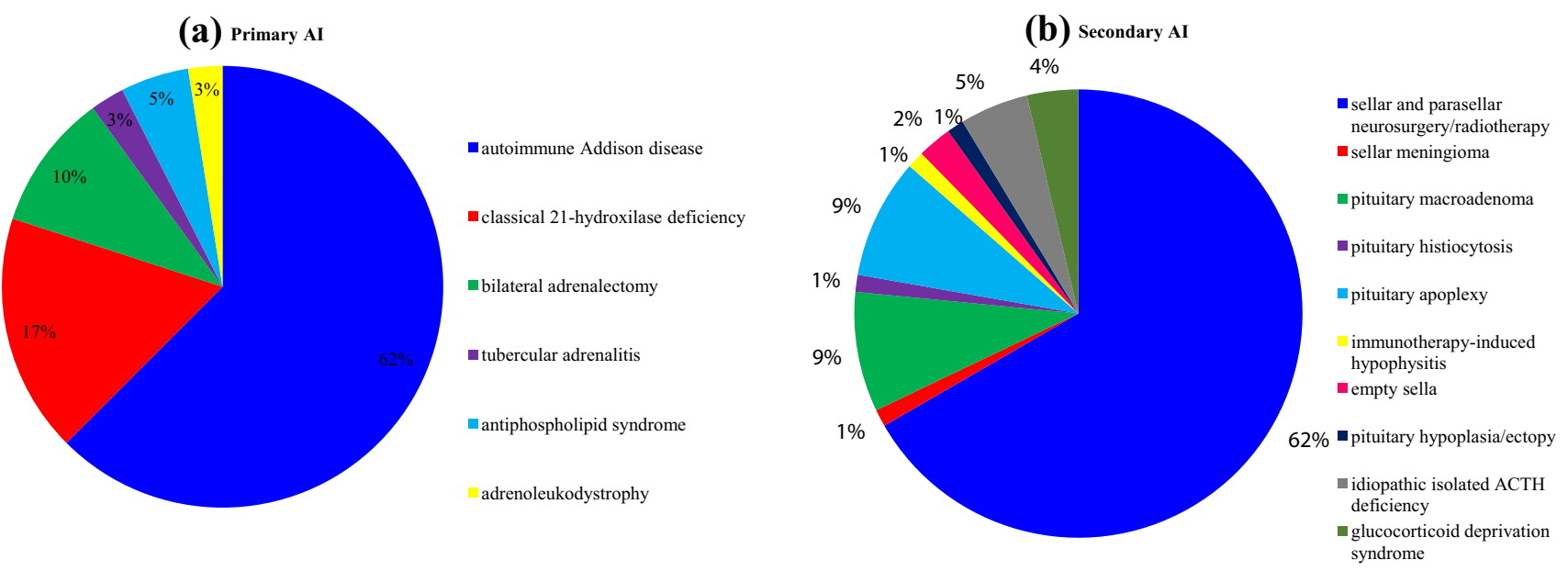

Fig. 3 Distribution of primary AI (a) and secondary AI (b) etiologies in the study cohort

Table 2 Results of CORTI-COVID, AddiQoL-30, and SF-36 questionnaires

\begin{tabular}{|c|c|c|c|c|}
\hline & All $(n=121)$ & Primary AI $(n=40)$ & Secondary AI $(n=81)$ & $p$ value \\
\hline CORTI-COVID & $17.7(16.9-18.6)$ & $17.8(16.3-19.2)$ & $17.7(16.7-18.7)$ & NS \\
\hline Global health concern & $3.8(3.6-4.0)$ & $3.7(3.3-4.1)$ & $3.9(3.6-4.1)$ & NS \\
\hline Personal health concern & $3.7(3.5-4.0)$ & $3.7(3.3-4.1)$ & $3.7(3.5-4.0)$ & NS \\
\hline Occupational concern & $2.6(2.3-2.8)$ & $2.8(2.3-3.2)$ & $2.5(2.1-2.8)$ & NS \\
\hline Economic concern & $3.8(3.6-4.1)$ & $3.9(3.6-4.3)$ & $3.8(3.5-4.1)$ & NS \\
\hline Social concern & $3.8(3.6-4.0)$ & $3.7(3.4-4.0)$ & $3.9(3.6-4.1)$ & NS \\
\hline ADDIQoL-30 & $130.6(126.0-135.3)$ & $130.9(122.2-139.6)$ & $130.5(125.0-136.1)$ & NS \\
\hline Fatigue & $33.0(31.4-34.6)$ & $33.8(30.7-37.0)$ & $32.6(30.8-34.5)$ & NS \\
\hline Symptoms & $41.3(39.8-42.8)$ & $40.8(38.2-43.3)$ & $41.6(39.7-43.4)$ & NS \\
\hline Emotions & $34.8(33.4-36.1)$ & $34.4(32.0-36.7)$ & $34.9(33.3-36.6)$ & NS \\
\hline Miscellaneous & $21.6(20.7-22.5)$ & $22 \cdot 0(20 \cdot 3-23 \cdot 6)$ & $21.4(20.3-22.5)$ & NS \\
\hline \multicolumn{5}{|l|}{ SF-36 } \\
\hline Physical functioning & $75.7(71.0-80.4) \%$ & $77.7(69.3-86.0) \%$ & $74.7(68.9-80.5) \%$ & NS \\
\hline Role limitation due to physical health & $66.5(59.3-73.8) \%$ & $65.0(52.2-77.8) \%$ & $67.3(58.3-76.3) \%$ & NS \\
\hline Role limitation due to emotional problems & $66.1(58.6-73.7) \%$ & $62.5(49.5-75.5) \%$ & $67.9(58.5-77.3) \%$ & NS \\
\hline Energy/fatigue & $58.7(54.6-62.7) \%$ & $57.4(49.6-65.1) \%$ & $59.3(54.5-64.1) \%$ & NS \\
\hline Emotional wellbeing & $66.9(63.3-70.5) \%$ & $65.4(59.0-71.8) \%$ & $67.6(63.2-72.0) \%$ & NS \\
\hline Social functioning & $66.6(62.2-71.0) \%$ & $66.6(58.5-74.6) \%$ & $66.7(61.3-72.0) \%$ & NS \\
\hline Physical pain & $71.6(66.7-76.4) \%$ & $71.1(62.1-80.1) \%$ & $71.8(65.9-77.6) \%$ & NS \\
\hline General health perception & $57.7(53.4-62.0) \%$ & $60.6(52.1-69.2) \%$ & $56.2(51.3-6.11) \%$ & NS \\
\hline
\end{tabular}

Data are expressed as mean $(95 \% \mathrm{CI})$

NS not statistically significant

except for autoimmune diseases (67\% vs $22 \%, p=0.029$; unconfirmed difference when selecting primary AI).

COVID-19 occurred only in one ( $0.8 \%$ prevalence) 48-year-old woman who developed primary AI in 1997, after bilateral adrenalectomy for Cushing's Syndrome due to Primary Pigmented Nodular Adrenocortical Disease (PPNAD). In February 2020, she had working contacts with a COVID-19 subject, who then died for respiratory distress. As symptoms started, the woman promptly adjusted glucocorticoid replacement and added nimesulide, with benefit. Dyspnea, chest and muscle pain, fever $\left(39.8^{\circ} \mathrm{C}\right)$, asthenia and vomiting lasted three days, without requiring hospitalization. Her CORTI-COVID score was 12 (global health $=3$, personal health $=1$, occupational consequences $=3$, economic impact $=2$, social consequences $=3$ ). Despite the presence of familial clusters in 
almost 5\%, COVID-19 was not reported among alive secondary AI participants. However, one of the ten patients excluded due to death (Fig. 1) was a 69-year-old woman with cognitive disorders and hypopituitarism arisen after surgery and subsequent radiotherapy for acromegaly. She died during the pandemic from bilateral pneumonia, which was not associated with SARS-CoV-2, according to her official death certificate. Close friends and colleagues were hit by COVID-19 in 23\% patients (nine with primary and 19 with secondary AI).

Occupations were distributed as shown in Supplemental Fig. 1. Lockdown-induced occupational changes involved $43 \%$ participants (24 primary AI and 28 secondary AI): the most frequent working restrictions were layoff (14\%), smart working (7.4\%) and withdrawal (6.6\%). Occupational concern was significantly higher in patients affected by working restrictions [3.2 (95\% CI 2.9-3.6) vs 2.0 (95\% CI 1.7-2.4), $\mathrm{p}<0.0001]$.

Participants were asked to choose which of the following sentences ("hashtags", \#) best represented their mood and thoughts: \#1 "let's hold on" (28\%), \#2 "everything will be fine" (11\%), \#3 "stay home" (36\%), \#4 "distant but joint" (17\%), \#5 "Italy does not stop" (8\%). A higher social worry was significantly associated to \#1 and \#3 $(p=0.039)$, as was the absence of working restrictions $(p=0.020)$; gender, age and glucocorticoid dose did not influence the choice. The COVID-19 patient chose \#2.

\section{ADDIQoL-30}

The results of ADDIQoL-30 questionnaire are described in Table 2. All domain-specific scores strongly correlated $(\rho=0.799 \div 0.932, p<0.0001)$ with ADDIQoL-30, which was influenced most by the domain "Fatigue".

Total and domain-specific ADDIQoL scores were similar between primary and secondary AI, regardless of age and glucocorticoid daily dose. However, a gender difference was highlighted for both total ADDIQoL and the domain "Symptoms", where males' QoL was better than females' ( $p=0.029$ and 0.005 , respectively; Supplemental Table 1). Non-diabetic patients had a significantly superior QoL than diabetic ones; also, a history of ischemic heart disease was associated with a worse QoL.

A moderate, negative correlation was found between ADDIQoL-30 and the degree of concern for the pandemic expressed by the CORTI-COVID score $(\rho=-0.474, p<0.0001)$. Moreover, all four ADDIQoL domains moderately correlated with the CORTICOVID score $(\rho=-0.350 \div-0.482, p<0.0001)$, as well as all five CORTI-COVID items correlated with ADDIQoL-30 $(\rho=-0.193 \div-0.421, p<0.035)$. The COVID-19 patient totalized an ADDIQoL-30 of 166/180
("Fatigue" = 44, "Symptoms" = 51, "Emotions" = 44, "Miscellaneous"=27).

\section{SF-36}

Table 2 illustrates patients' SF-36. All results were significantly associated, but the strongest correlation was found between the domains "Energy/fatigue" and "Emotional wellbeing" $(\rho=0.786, p<0.0001)$; "Energy/fatigue" was the most contributing domain to the "General health perception" $(\rho=0.700, p<0.0001)$.

SF-36 results were similar between primary and secondary AI. Males had a slightly better health perception than females, which became significant for the domains "Energy/ fatigue" and "Emotional wellbeing" ( $p=0.030$ and 0.044 , respectively; Supplemental Table 2). Elder participants scored worse in "Physical functioning" (correlation with age $\rho=-0.260, p=0.004$ ), whereas patients using higher glucocorticoid doses scored better in "Physical pain" $(\rho=0.212$, $p=0.020$ ). Diabetes mellitus was the comorbidity with the largest negative impact on QoL; cardiovascular diseases/risk factors impaired the "Social functioning" and, as autoimmune disorders, the "Role limitation due to physical health".

The correlation between SF-36 and ADDIQoL-30 was strong, particularly in detecting fatigue $(\rho=0.802$, $p<0.0001)$ and emotional aspects $(\rho=0.814, p<0.0001)$. The domains "Energy/fatigue", "Emotional wellbeing" and "General health perception" showed the strongest correlations with ADDIQoL-30 $(\rho=0.836,0.772$ and 0.734 , respectively; $p<0.0001$ ).

Almost all SF-36 domains were inversely correlated with the CORTI-COVID items, the strongest correlation existing between total CORTI-COVID and "General health perception" $(\rho=-0.425 p<0.0001)$. Participants with a higher "General health perception" preferred optimistic hashtags $(\# 4, \# 5)$ over resilient ones $(p=0.035)$. The COVID-19 patient perceived a good health-related QoL, scoring 100\% in most domains ("Energy/fatigue" $=70 \%$, "Emotional wellbeing" $=64 \%$, "General health perception" $=90 \%$ ).

\section{Primary vs Secondary Al (Table 2)}

Concern for personal health most influenced the final score in primary $\mathrm{AI}(\rho=0.888, p<0.0001)$. Concern for economy most impacted the secondary AI final score $(\rho=0.854$, $p<0.0001)$, which was inversely correlated with age ( $\rho=-0.236, p=0.043)$; the items "occupation" $(\rho=-0.385$, $p=0.001)$ and "economy" $(\rho=-0.286, p=0.018)$ showed the same correlation pattern. Gender, geographical distribution and glucocorticoid replacement did not influence the separate scores. The presence of comorbidities played a significant role only in secondary AI: hypertensive patients were more worried for COVID-19-related global 
health $(p=0.016)$ and less for occupational consequences $(p=0.006)$; patients with autoimmune diseases were more afraid of personal health consequences $(p=0.008)$. Secondary AI patients with autoimmune diseases were also more worried for COVID-19-related global health $(p=0.036)$, social consequences $(p=0.010)$, and totalized a higher CORTI-COVID score than their counterpart (20.7 (95\% CI 18.2-23.2) vs 17.4 (95\% CI 16.3-18.4), $p=0.034$ ). The degree of occupational concern persisted higher for people affected by working restrictions, which involved $60 \%$ of primary AI (3.1 (95\% CI 2.7-3.6) vs 2.2 (95\% CI 1.4-3.0), $p=0.022)$ and $35 \%$ of secondary AI (3.3 (95\% CI 2.8-3.9) vs 2.0 (95\% CI 1.6-2.4), $p<0.0001$ ) patients. Patients with primary AI showed a gender difference in the chosen hashtag (\#5 for males, \#1 and \#3 for females, $p=0.023$ ); moreover, patients with primary AI who continued to work without restrictive measures chose \#1, while \#3 and \#5 were preferred by primary AI patients under working restrictions $(p=0.024)$. Regardless of AI etiology, age and glucocorticoid dose correlated with no specific hashtags.

Distribution of ADDIQoL-30 scores according to AI etiology is shown in Fig. 4. ADDIQoL-30 was similar between groups: nevertheless, the domain "Fatigue" was the most impacting the ADDIQoL-30 of patients with primary AI $(\rho=0.958, p<0.0001)$, whereas "Symptoms" influenced secondary AI ADDIQoL-30 most $(\rho=0.857$, $p<0.0001)$. A gender difference favoring males emerged in the secondary AI group for ADDIQoL-30, as well as for most domains ( $p=0.001-0.031$; Supplemental Table 1); no correlations were found concerning age and glucocorticoid daily dose. In general, the presence of comorbidities was associated to a worse QoL, as shown in Supplemental Table 1 . In both subgroups, ADDIQoL-30 was moderately, inversely correlated with total CORTI-COVID score $(\rho=-0.583$ and -0.427 for primary and secondary AI, respectively; $p<0.0001)$ and all partials $(p<0.025)$ but "occupational concern".

Also SF-36 results were similar between primary and secondary AI patients. "General health perception" in primary AI was most influenced by the domain "Social functioning" $(\rho=0.671, p<0.0001)$, whereas in secondary AI the most impacting domain was "Energy/Fatigue" $(\rho=0.743$, $p<0.0001)$. The gender difference favoring males confirmed only for the secondary AI group ( $p=0.013-0.032$, Supplemental Table 2). Similarly, the relationship between glucocorticoid dose and Physical pain was found only in secondary AI $(\rho=0.302, p=0.006)$. Only elder primary AI patients had worse Physical functioning- and Physical painrelated QoL scores $(\rho=-0.341, p=0.031$ and $\rho=-0.314$ $p=0.048$, respectively).

The presence of diabetes mellitus and overweight/obesity impaired QoL of both groups, however involving different SF-36 domains (Supplemental Table 2). The correlation between SF-36 and ADDIQoL-30 persisted good ( $\rho=0.620-0.842$ for primary AI and $\rho=0.584-0.822$ for secondary AI, $p<0.0001$ ), especially concerning "Energy/
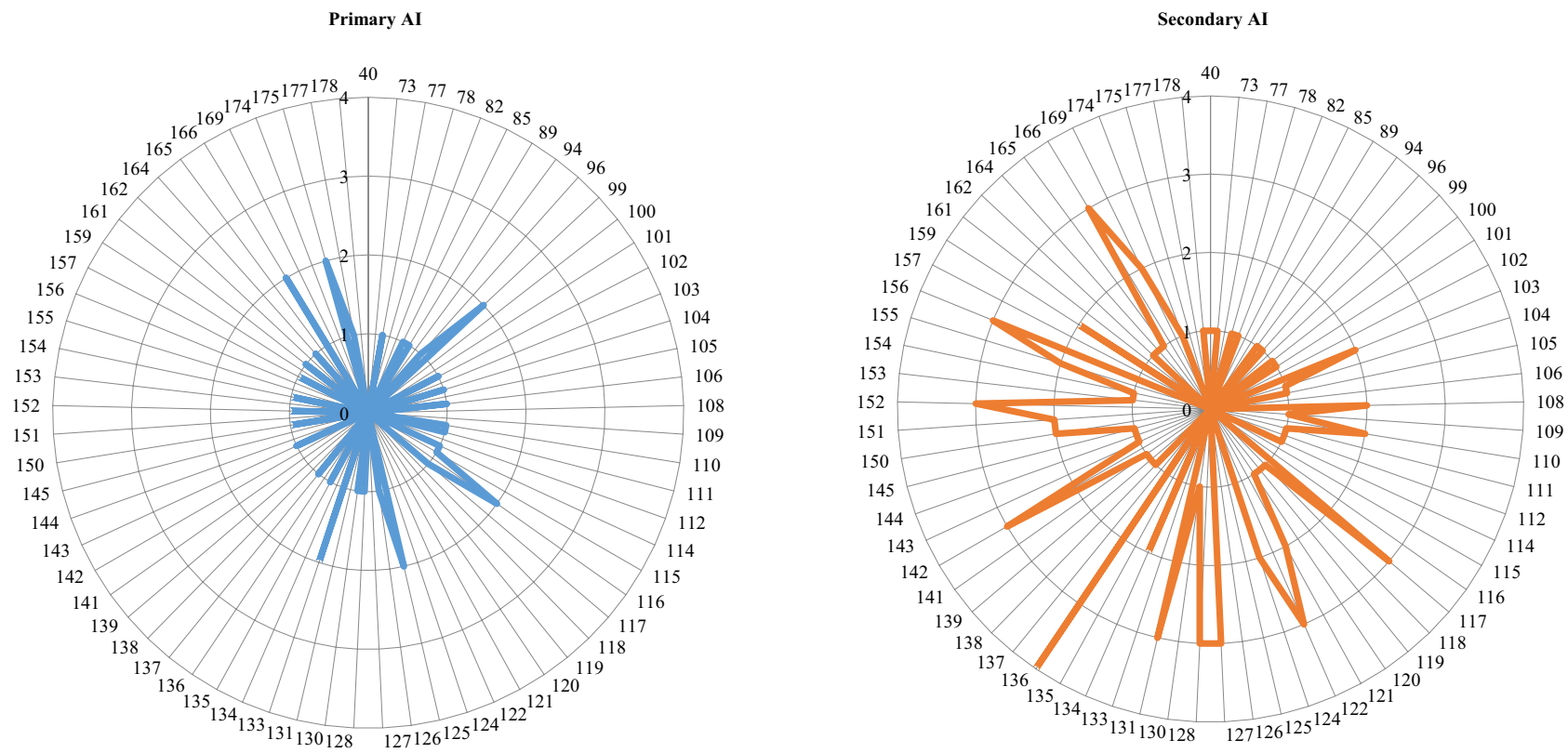

Fig. 4 AddiQoL-30scores totalized by patients with primary (blue lines) and secondary AI (red lines). Extension of colored lines indicates the number of patients (concentric circles) totalizing each score 
fatigue" and "Emotional wellbeing"; moreover, the whole cohort findings on the correlation between SF-36 and CORTI-COVID were confirmed.

\section{Discussion}

This is the first study evaluating the impact of SARSCoV-2 pandemic on psychophysical stress of patients with $\mathrm{AI}$ in clinical practice.

Due to the current lockdown dispositions, 40 patients with primary $\mathrm{AI}$ and 81 patients with secondary AI underwent a telematics interview aimed at exploring first the prevalence, clinical features and outcome of COVID-19. Only a woman with postsurgical primary AI was infected with SARS-CoV-2; as confirmed by her questionnaire results, she promptly applied the "sick days" rules managing the infection with confidence and did not experience impairments in QoL or health status. Considering that the current seroprevalence of SARS-CoV-2 antibodies in Italian population is $2.5 \%$, as stated by the latest National Institute of Statistics (ISTAT) SARS-CoV-2 seroprevalence survey [19], and despite the high prevalence of autoimmune disease in the study cohort, our AI patients seem not at particular risk of COVID-19 (prevalence 0.8\%). This seems quite surprising for several reasons: first, AI patients are notably susceptible to infections due to their inefficient innate immune system and hypothalamus-pituitary-adrenal axis activation in response to stressors [20]; second, worse outcomes may be expected in COVID-19 pneumonia, where the "cytokine storm" can target the adrenal gland with disruption of residual function [21]; third, it must be considered that most of our patients live in Marche Region, which was included among the "red areas" for weeks due to the elevated contagion rates.

A second investigation topic was the occurrence of adrenal crises and their possible association with concomitant COVID-19 and/or pandemic-related psychophysical stress. In a two-year follow-up study on 423 patients with AI, Hahner et al. observed 46 adrenal crises: of them, $14(30 \%)$ were triggered by emotional factors [7]. Here, although the whole cohort was exposed simultaneously to an unexpected emotional stress, no adrenal crises were reported. However, six of the 14 patients who required glucocorticoid stress doses in the previous two months (i.e. $5 \%$ of study participants) identified in the pandemic-related emotional stress the reason for the up-titration. This, along with the reduced physical stress due to social distancing, could have had a role in preventing adrenal crises in our cohort and confirms the central importance of health education in AI. It must be noticed that all primary AI patients who needed glucocorticoid stress doses were females, which suggests that women with primary AI may be more conscious of their disease, have more anxiety or less adaptive capacity to psychophysical stress than men. This is in line with existing data on the correlation between female gender and the risk of overtreatment in patients with AI [22].

To assess the emotional impact of the pandemic-induced lockdown and social distancing we asked our patients to fill out telematically the "CORTI-COVID", a purpose-built questionnaire exploring patients' concern about COVID19-related general health, AI-specific health, occupational consequences, economic impact, and social implications. Most available patients joined the initiative with enthusiasm, appreciating the opportunity of a remote, but close contact with their dedicated clinicians during such a difficult moment. Globally, our patients' degree of concern was high (mean CORTI-COVID score 17.7, 95\% CI 16.9-18.6), without differences related to demography, replacement therapy, or AI etiology (Fig. 5). However, secondary AI patients were more worried about the economic impact of the pandemic, whereas primary AI patients feared for their personal health most ( $\rho=0.854$ and 0.888 , respectively; $p<0.0001)$. This finding is of crucial importance since patients with primary AI seem more aware of their fragility and serious health risks than patients with secondary AI, where the involvement of other pituitary deficiencies and related comorbidities often diverts their attention from the importance of preserving adrenal function. Especially in secondary AI patients, indeed, the presence of comorbidities, above all hypertension, was associated with worse CORTI-COVID scores. Considering that both hypertension and overweight/ obesity are negative prognostic factors for ARDS and death in COVID-19, particularly in elderly men [23], this is not surprising, since in our secondary AI patients hypertension was more prevalent in males, often concurring with overweight/obesity and old age.

The CORTI-COVID questionnaire well characterized the emotional impact of the pandemic for several reasons: first, it is targeted to this study purpose; second, even if a control group for the validation is lacking, the simultaneous completion by a large cohort of patients, who were all exposed to the same stressor, empowers its validity; third, its results correlated with those of ADDIQoL-30 and SF-36, two of the most largely used questionnaires to assess the self-reported health-related QoL both in AI and in the general population [5, 17, 24], making CORTICOVID a very reliable tool. With respect to QoL and health status, patients were in a general state of wellbeing, but reported a widespread concern, which involved personal health and occupational issues in the younger, economic and social aspects in the elder. Psychological impact due to working restrictions and economic damage was certainly strong (21\% patients underwent layoff or withdrawal) but, in contrast to Hahner's previous findings [7], it fortunately did not trigger any adrenal crises. 
Fig. 5 Percentage distribution of the total CORTI-COVID score totalized by patients with primary (blue bars) and secondary A
Total CORTI-COVID score

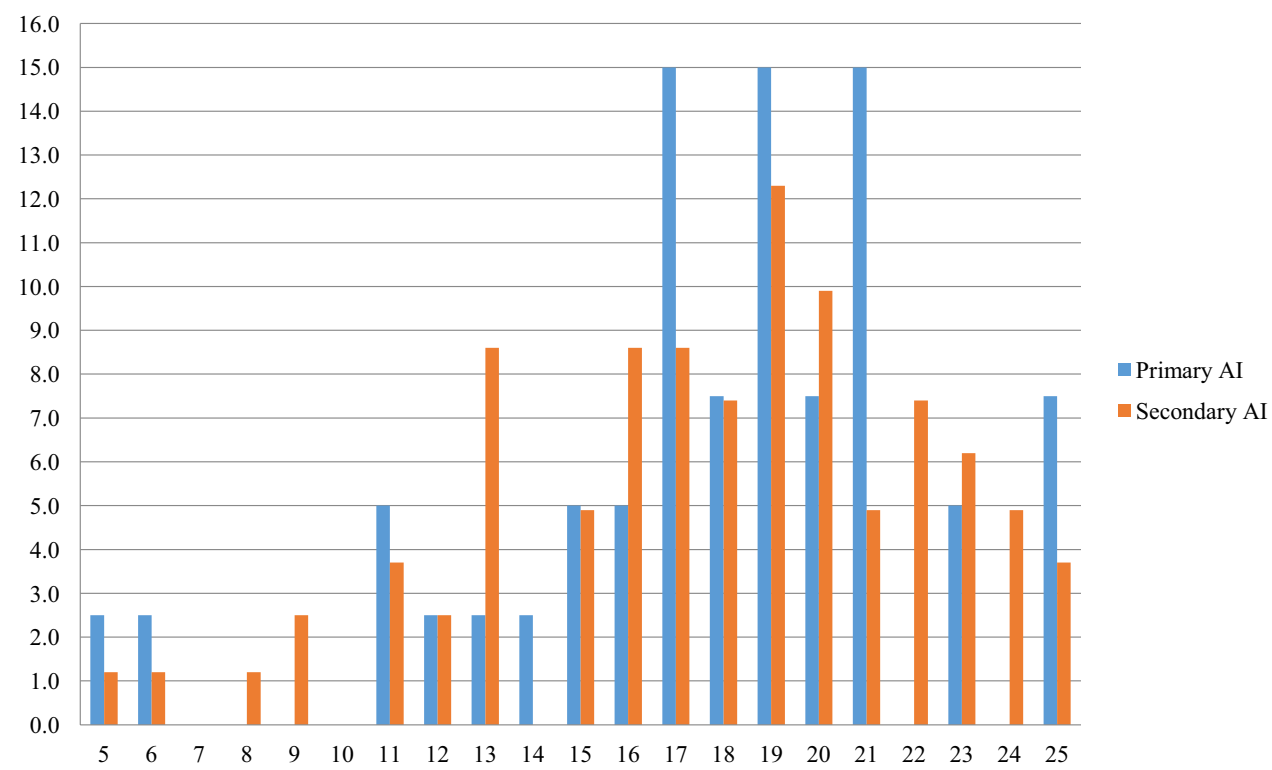

Patients' adaptive capacity to this unconventional situation was good, as attested by the preferential choice of resilient hashtags (\#1, \#3, \#4) over-optimistic ones (\#2, \#5) by more socially worried and less occupationally restricted participants. The perception of danger and discomfort was higher in patients with comorbidities, leading to significantly lower QoL and health status. Again, this is not surprising if we consider the negative prognostic impact of diabetes mellitus and hypertension on COVID-19 [23]; yet, an unexpected finding is the pejorative impact of autoimmune diseases on QoL and health perception not only in primary AI but also in secondary AI patients. However, even after separating the two etiologic subgroups, the results of ADDIQoL-30 and SF-36 persisted good and correlated well: this is interesting since ADDIQoL-30 has been validated only for primary AI patients [16]; here, we show its reliability also in assessing QoL of patients with secondary AI. Of note, the self-reported QoL and health status were superior in our cohort than in previous cohorts [15, 16], further confirming the importance of ensuring adequate, tailored replacement regimens and unceasing education to all AI patients.

As previously anticipated, the main limitation of this study is that the CORTI-COVID questionnaire was not administered to healthy individuals, making it difficult to evaluate whether the observed results, particularly occupational aspects, are specific for AI or are not dependent on it: this was due to the current pandemic situation and its adjoining urgent needs. We believe this lacking does not change the quality of our findings (comparison between primary and secondary AI), but a proper control group, as well as a validated English or multi-language version (for original Italian version see Supplemental Fig. 2) of the CORTI-COVID questionnaire would be certainly needed for future research.

\section{Conclusions}

Provided that educational efforts are made to prevent and treat acute events, both primary and secondary AI patients seem not at particular risk of COVID-19. However, the pandemic is exposing AI patients to a strong emotional burden, which is assessed reliably through the novel, purpose-built CORTI-COVID score. Even in unconventionally stressful situations, as this pandemic is, educated AI patients preserve good QoL and health perception.

Acknowledgements The authors wish to thank all study participants for their enthusiastic adhesion to this initiative in such a difficult time.

Author contributions NA and MFC contributed equally to the manuscript. MM and GA designed the study. MM, NA, MFC, GG, and AC recruited patients. NA, MFC, GG, and AC collected the data. MM, GS, and GA analysed the data. MM, GS, and GA interpreted the data. MM, NA, MFC, and GA drafted the manuscript. MM, NA, MFC, and GA created figures. MM, NA, MFC, GG, AC, GS, and GA critically revised the manuscript for important intellectual content.

Funding This work was supported by the MIUR (Ministero dell'Istruzione dell'Università e della Ricerca, grant number HRTZYA_005, 2017).

Data availability The datasets generated and analysed are available from the corresponding author on reasonable request. 


\section{Compliance with ethical standards}

Conflict of interest The authors declare that they have no conflict of interest.

Ethical approval The study was conducted in accordance with the ethical standards for research involving human participants. Protocol approval for evaluation of psychophysical stress in patients with AI was obtained from the Ethical Committee of the University Hospital of Ancona.

Informed consent Informed consent was obtained from all individual participants included in the study.

\section{References}

1. Bornstein SR, Allolio B, Arlt W et al (2016) Diagnosis and treatment of primary adrenal insufficiency: an endocrine society clinical practice guideline. J Clin Endocrinol Metab 101(2):364-389. https://doi.org/10.1210/jc.2015-1710

2. Fleseriu M, Hashim IA, Karavitaki N et al (2016) Hormonal replacement in hypopituitarism in adults: an endocrine society clinical practice guideline. J Clin Endocrinol Metab 101(11):3888-3921. https://doi.org/10.1210/jc.2016-2118

3. Bergthorsdottir R, Leonsson-Zachrisson M, Odén A, Johannsson G (2006) Premature mortality in patients with Addison's disease: a population-based study. J Clin Endocrinol Metab 91(12):48494853. https://doi.org/10.1210/jc.2006-0076

4. Sherlock M, Ayuk J, Tomlinson JW et al (2010) Mortality in patients with pituitary disease. Endocr Rev 31(3):301-342. https ://doi.org/10.1210/er.2009-0033

5. Hahner S, Loeffler M, Fassnacht $M$ et al (2007) Impaired subjective health status in 256 patients with adrenal insufficiency on standard therapy based on cross-sectional analysis. J Clin Endocrinol Metab 92(10):3912-3922. https://doi.org/10.1210/ jc. 2007-0685

6. Allolio B (2015) Extensive expertise in endocrinology. Adrenal crisis. Eur J Endocrinol. 172(3):R115-R124. https://doi. org/10.1530/EJE-14-0824

7. Hahner S, Spinnler C, Fassnacht M et al (2015) High incidence of adrenal crisis in educated patients with chronic adrenal insufficiency: a prospective study. J Clin Endocrinol Metab 100(2):407416. https://doi.org/10.1210/jc.2014-3191

8. Wang D, Hu B, Hu C et al (2020) Clinical characteristics of 138 hospitalized patients with 2019 novel coronavirus-infected pneumonia in Wuhan, China. JAMA 323(11):1061-1069. https://doi. org/10.1001/jama.2020.1585

9. Huang C, Wang Y, Li X et al (2020) Clinical features of patients infected with 2019 novel coronavirus in Wuhan, China. Lancet 395(10223):497-506. https://doi.org/10.1016/S0140 $-6736(20) 30183-5$

10. Wu Z, McGoogan JM (2020) Characteristics of and important lessons from the coronavirus disease 2019 (COVID-19) outbreak in China: summary of a report of 72,314 cases from the Chinese Center for Disease Control and Prevention. JAMA. https://doi. org/10.1001/jama.2020.2648(Online ahead of print)

11. Onder G, Rezza G, Brusaferro S (2020) Case-fatality rate and characteristics of patients dying in relation to COVID-19 in Italy.
JAMA. https://doi.org/10.1001/jama.2020.4683(Online ahead of print)

12. Baud D, Qi X, Nielsen-Saines K, Musso D, Pomar L, Favre G (2020) Real estimates of mortality following COVID-19 infection. Lancet Infect Dis 20(7):773. https://doi.org/10.1016/S1473 -3099(20)30195-X

13. Isidori AM, Arnaldi G, Boscaro $M$ et al (2020) COVID-19 infection and glucocorticoids: update from the Italian Society of Endocrinology expert opinion on steroid replacement in adrenal insufficiency. J Endocrinol Invest 43(8):1141-1147. https://doi. org/10.1007/s40618-020-01266-w

14. Arlt W, Baldeweg SE, Pearce SHS, Simpson HL (2020) Endocrinology in the time of COVID-19: management of adrenal insufficiency. Eur J Endocrinol 183(1):G25-G32. https://doi. org/10.1530/EJE-20-0361

15. Løvås K, Curran S, Oksnes M, Husebye ES, Huppert FA, Chatterjee VK (2010) Development of a disease-specific quality of life questionnaire in Addison's disease. J Clin Endocrinol Metab 95(2):545-551. https://doi.org/10.1210/jc.2009-1711

16. Øksnes M, Bensing S, Hulting AL et al (2012) Quality of life in European patients with Addison's disease: validity of the disease-specific questionnaire AddiQoL. J Clin Endocrinol Metab 97(2):568-576. https://doi.org/10.1210/jc.2011-1901

17. Meyer G, Koch M, Herrmann E, Bojunga J, Badenhoop K (2018) Longitudinal AddiQoL scores may identify higher risk for adrenal crises in Addison's disease. Endocrine 60(2):355-361. https://doi. org/10.1007/s12020-017-1513-0

18. Ware JE Jr, Sherbourne CD (1992) The MOS 36-item short-form health survey (SF-36). I. Conceptual framework and item selection. Med Care 30(6):473-483

19. https://www.slideshare.net/slideistat/primi-risultati-dellindaginedi-sieroprevalenza-sarscov2. Accessed on 3 Aug 2020

20. Isidori AM, Venneri MA, Graziadio C et al (2018) Effect of oncedaily, modified-release hydrocortisone versus standard glucocorticoid therapy on metabolism and innate immunity in patients with adrenal insufficiency (DREAM): a single-blind, randomised controlled trial. Lancet Diabetes Endocrinol 6(3):173-185. https ://doi.org/10.1016/S2213-8587(17)30398-4

21. Téblick A, Peeters B, Langouche L, Van den Berghe G (2019) Adrenal function and dysfunction in critically ill patients. Nat Rev Endocrinol 15(7):417-427. https://doi.org/10.1038/s4157 4-019-0185-7

22. Mazziotti G, Formenti AM, Frara S et al (2017) Management of endocrine disease: risk of overtreatment in patients with adrenal insufficiency: current and emerging aspects. Eur J Endocrinol 177(5):R231-R248. https://doi.org/10.1530/EJE-17-0154

23. Zhou F, Yu T, Du R et al (2020) Clinical course and risk factors for mortality of adult inpatients with COVID-19 in Wuhan, China: a retrospective cohort study. Lancet 395(10229):1054-1062. https ://doi.org/10.1016/S0140-6736(20)30566-3

24. Apolone G, Mosconi P (1998) The Italian SF-36 Health Survey: translation, validation and norming. J Clin Epidemiol 51(11):1025-1036. https://doi.org/10.1016/s0895-4356(98)00094 $-8$

Publisher's Note Springer Nature remains neutral with regard to jurisdictional claims in published maps and institutional affiliations. 